\title{
Carotid Artery Stenting in Right-sided Aortic Arch: A Case Report
}

\author{
Toshiyuki Ohtani,, Tomosato Yamazaki, ${ }^{2}$ Hiroya Ohtaki, ${ }^{1}$ Satoshi Nakata, ${ }_{1}^{1}$ Nobuo Sasaguchi, \\ Noriyuki Kato, ${ }^{2}$ Hideyuki Kurihara, ${ }^{1}$ and Makoto Sonobe ${ }^{2}$
}

\begin{abstract}
The present report describes a patient with pseudoocclusion of the left internal carotid artery accompanied by aortic anomalies consisting of right-sided aortic arch with aberrant left subclavian artery arising from Kommerell's diverticulum. Initial attempt of carotid artery stenting via the trans-femoral approach was unsuccessful because of low origin of the left common carotid artery. Therefore, carotid artery stenting (CAS) via the trans-brachial approach was successfully performed with distal balloon protection. Eight months later, the patient presented with restenosis of the left internal carotid artery, and CAS via the trans-brachial approach was performed again. CAS via the transbrachial approach should be considered when standard femoral access is relatively contraindicated due to aortic anomalies consisting of a right-sided aortic arch.
\end{abstract}

Keywords: aortic arch syndrome, internal carotid artery stenosis, stroke, carotid artery stenting

\section{Introduction}

Right-sided aortic arch is a rare congenital malformation occurring during embryologic development. The incidence of a right-sided aortic arch is approximately $0.1 \%$ in radiology and autopsy series. ${ }^{1,2)}$ Right-sided aortic arch results from persistence of the right fourth aortic arch and involution of the left fourth aortic arch, and details of the embryology of this region have been described. ${ }^{3)}$ A right-sided aortic arch with left aberrant subclavian artery is the most common type of this malformation. Kommerell's diverticulum most frequently accompanies this type of malformation and is the result of reabsorption of the left fourth aortic arch proximal to the origin of the left subclavian artery. ${ }^{3,4)}$ We treated a patient with pseudo-occlusion of the left internal carotid artery (ICA) accompanied by right-sided aortic arch with left aberrant subclavian artery arising from Kommerell's diverticulum. Our experience will provide useful information to guide neuroendovascular treatment of this condition.

\section{Case Report}

The patient was a 69-year-old man with a medical history of hypertension, diabetes mellitus, and hyperlipidemia. He was referred to our medical center at 2 weeks after the onset

${ }^{1}$ Department of Neurosurgery, National Hospital Organization, Takasaki General Medical Center, Takasaki, Gunma

${ }^{2}$ Department of Neurosurgery, National Hospital Organization, Mito Medical Center, Mito, Ibaraki

Received: January 8, 2015; Accepted: August 18, 2015 of transient aphasia and right hemiparesis due to acute brain infarction (Fig. 1A). Magnetic resonance angiography of the neck and the brain showed occlusion of the left ICA (Fig. 1B). Single photon emission tomography showed hemodynamic Type 2 stage based on Kuroda's classification. ${ }^{5)}$ Digital subtraction angiography showed anterograde string-like filling of the ICA beyond the carotid bifurcation and showed retrograde filling of the proximal ICA, extending up to the skull base (Fig. 1C, D). We confirmed acute brain infarction due to pseudo-occlusion of the left ICA. Aortography showed right-sided aortic arch, and computed tomography angiography was performed to evaluate an access route to the target lesion (Fig. 2A, B). The aortic arch was observed on the right side of the trachea and esophagus (Fig. 2C). Vessels from the right-sided aortic arch arose in the following order: left common carotid artery (CCA), right CCA, right subclavian artery, and left subclavian artery. The left subclavian artery originated at the junction between the distal aortic arch and the descending aorta located in the right chest. The origin of
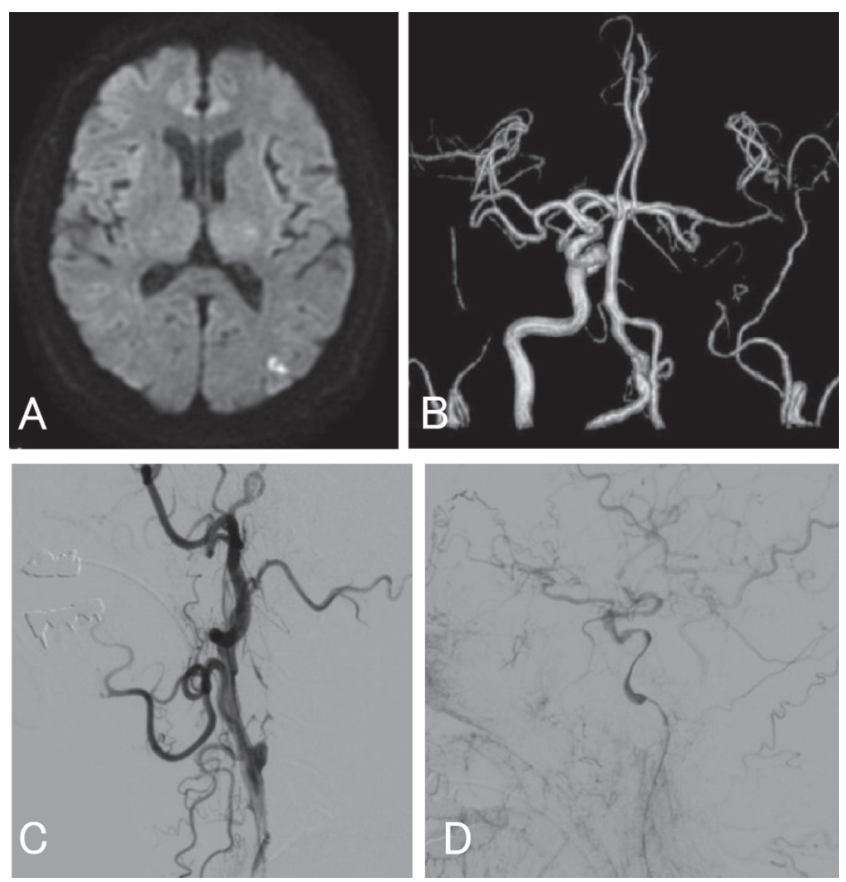

Fig. 1 A: Diffusion-weighted magnetic resonance imaging shows left middle cerebral artery-posterior cerebral artery border zone infarction. B: Magnetic resonance angiography reveals occlusion of the left internal carotid artery. C, D: Lateral views of the left carotid angiogram reveal pseudo-occlusion of the left internal carotid artery. 

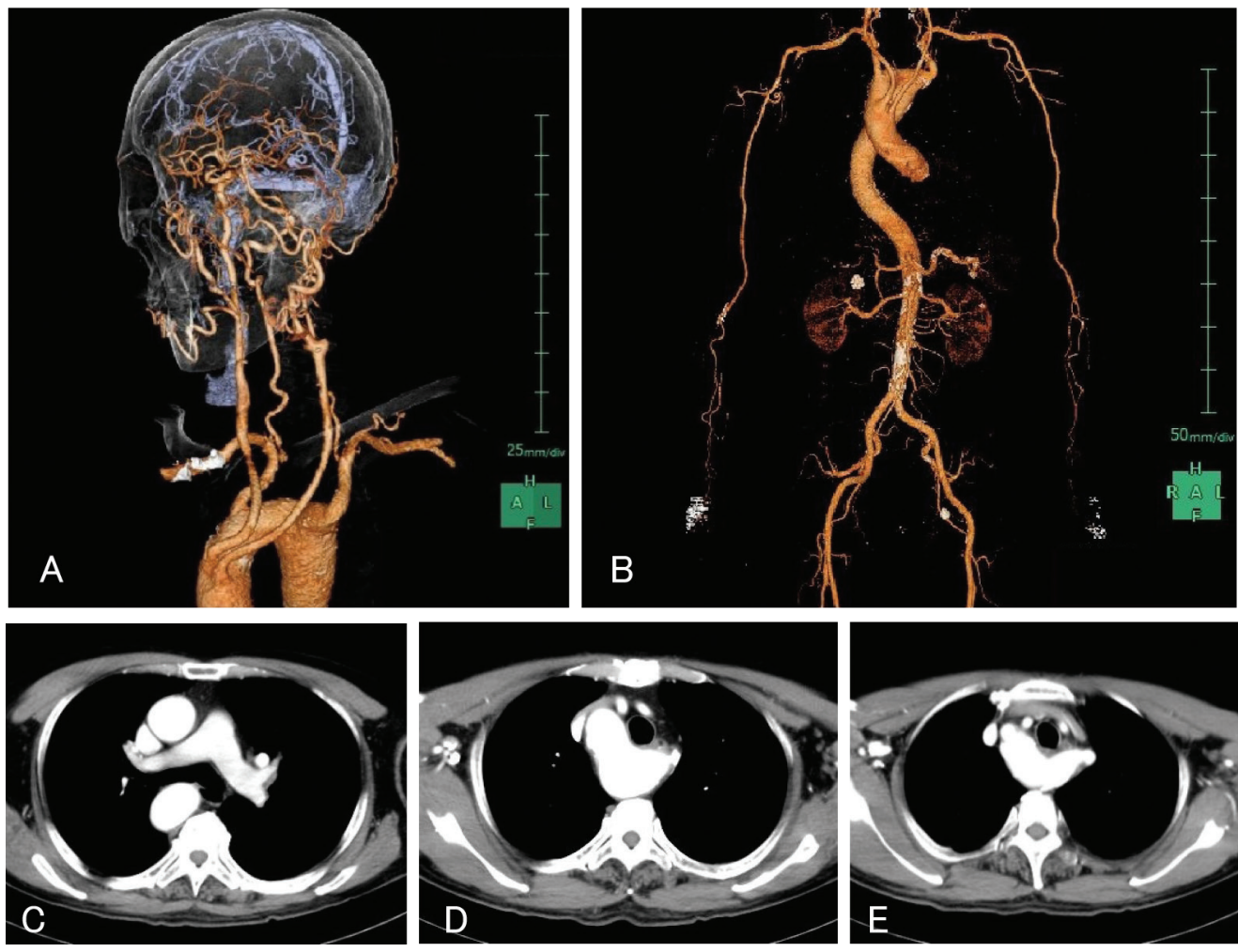

Fig. 2 A, B: Computed tomography angiograms show a right-sided aortic arch and the aortic arch branches in the following order: left common carotid, right common carotid, right subclavian, and left subclavian arteries (from proximal to distal). B: The right-sided descending thoracic aorta is seen. C, D: Computerized axial tomography shows the aortic arch passing over the right main stem bronchus. C-E: The aortic arch is observed on the right side of the trachea and the esophagus. the left subclavian artery was aneurysmal (i.e., Kommerell's diverticulum; Fig. 2D, E). The left vertebral artery arose from the left subclavian artery. The left CCA arose from the lower part of the ascending aorta and passed over the trachea and the esophagus to the left side. We confirmed the presence of a right-sided aortic arch with an aberrant left subclavian artery.

After informed consent was obtained, we performed carotid artery stenting (CAS) for symptomatic left ICA pseudo-occlusion under general anesthesia (Figs. 3, 4). A 9-Fr sheath was inserted into the right femoral artery. A 9-Fr occlusion balloon-guiding catheter (OPTIMO; Tokai Medical Products, Inc., Aichi) was navigated into the descending thoracic aorta on the right side of the trachea using the coaxial method (Fig. 3A, B). After cannulation of the left CCA with the tip of the inner catheter (Fig. 3C), we carefully advanced a coaxially inserted 0.035 -inch diameter guidewire into the left maxillary artery. We tried to advance the inner catheter to the left external carotid artery, but failed because the inner catheter fell into the ascending aorta. Subsequently, CAS was performed via the trans-brachial approach. A 6-Fr guiding sheath (Axcelguide; Medikit Co. Ltd., Tokyo) was advanced into the brachiocephalic artery using the coaxial method. After cannulation of the left CCA with the tip of a 6-Fr Simmons-type inner catheter (SY-2 125 cm: Medikit, Tokyon), we inserted a 0.035-inch diameter guidewire (Radifocus standard type $300 \mathrm{~cm}$; Terumo Corporation, Tokyo) into the third portion of left maxillary artery. We carefully placed the 6-Fr Simmons-type inner catheter into the left CCA and then exchanged the guidewire for a half-stiff 0.035-inch diameter guidewire (Radifocus half-stiff type
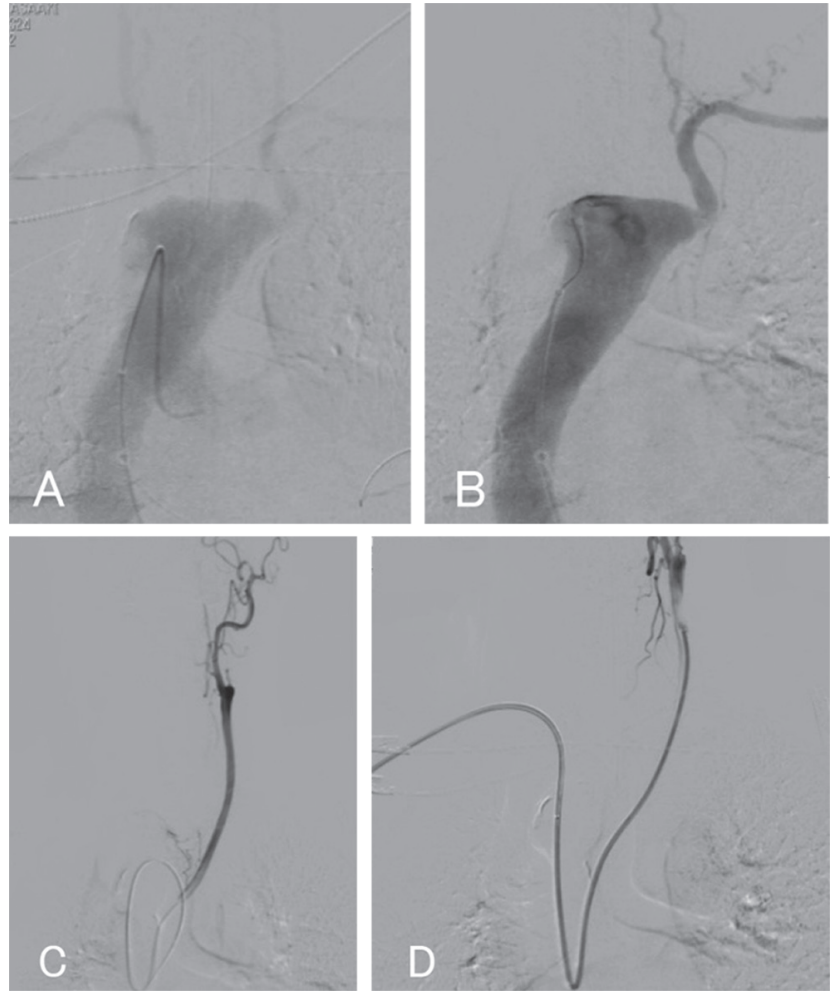

Fig. 3 A: Aortography shows a right-sided aortic arch. B: Kommerell's diverticulum and the left vertebral artery originated from an aberrant left subclavian artery. C: Failure to cannulate the left common carotid artery with a 9-Fr occlusion balloon-guiding catheter via the trans-femoral approach. D: Success in advancing the 6-Fr guiding sheath to the left common carotid artery via the trans-brachial approach. 

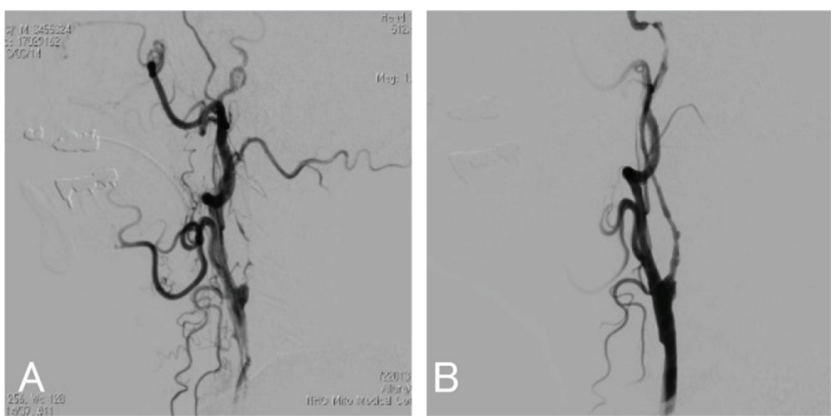

Fig. 4 Lateral view of the left carotid angiograms before (A) and immediately after (B) initial carotid artery stenting reveals an improved stenotic lesion.

$300 \mathrm{~cm}$; Terumo, Tokyo). We advanced the 6-Fr guiding sheath over the inner catheter and turned the 6-Fr guiding sheath toward the left CCA just above the aortic valve, and subsequently succeeded in advancing the 6-Fr guiding sheath to the left CCA (Fig. 3D). A Guardwire ${ }^{\circledR}$ (Medtronic Inc., Santa Rosa, California, USA) distal-protection balloon was positioned within the left ICA, distal to the stenosis. Predilatation was performed using a 3-mm diameter balloon (Ultrasoft-SV, Boston Scientific Corporation, Natick, Massachusetts, USA). Stent deployment was then performed using an $8 \times 21 \mathrm{~mm}$ self-expanding Carotid Wallstent (Boston Scientific Corp, Massachusetts). Digital subtraction angiography immediately after CAS showed successful stent implantation, and antegrade blood flow of the left ICA was restored (Fig. 4).

Eight months later, restenosis of the left ICA occurred, and CAS was again performed via the trans-brachial approach. We placed a $9 \times 60 \mathrm{~mm}$ self-expanding Protégé stent (ev3 Inc., Plymouth, Minnesota, USA) in the distal part of the Carotid Wallstent. The blood flow through the left ICA was significantly improved. The postoperative course was uneventful. Diffusion-weighted images on the day after the procedure showed a few thromboembolic lesions without any neurological deficits. The patient was discharged home at 5 days after the procedure.

\section{Discussion}

A right-sided aortic arch is the result of abnormal organogenesis of the primitive aortic arches. Previous reports described three main types of right-sided aortic arches: type I, with mirror-image branching of the major arteries; type II, with an aberrant left subclavian artery; and type III, with isolation of the left subclavian artery where the subclavian artery is connected to the pulmonary artery through the ductus arteriosus. ${ }^{3,46)}$ Type II is most common type of this abnormality and is associated with an aberrant left subclavian artery arising either from the last branch of the right-sided aortic arch or from an aortic diverticulum. This diverticulum is termed Kommerell's diverticulum and is a remnant of the left dorsal aortic arch. ${ }^{3)}$ This occurs as a result of reabsorption of the left fourth aortic arch proximal to the origin of the left subclavian artery. ${ }^{7,8)}$ The right-sided aortic arch passes over the right main stem bronchus to the right of the trachea and esophagus. It is usually associated with involution of the left dorsal aorta and persistence of the right dorsal aorta, causing the descending thoracic aorta to be located in the right thorax. If, instead, the right dorsal aorta disappears, the right-sided aortic arch passes behind the esophagus to join the left dorsal aorta, and the thoracic aorta descends in the left chest. ${ }^{3,9)}$

If the catheter moves between the center and right side in the chest during cerebral angiography performed via the femoral artery, the examiner should think about the possibility that this aortic arch is a right-sided aortic arch from the motion of a catheter. In our case, the main four vessels arose independently in the following order: the first, running toward the left cervical region in front of the trachea, was the left CCA (left innominate artery); the second, ascending to the right of the trachea, was the right CCA; the third was right subclavian artery; and the last was aberrant left subclavian artery. Our case involved a type II right-sided aortic arch with an aberrant left subclavian artery. It is very important that we understand anatomical variations and the characteristics of right-sided aortic arch, as examination of the cerebral angiography should be carried out with considerable care due to these exceptional anatomical anomalies.

To the best of our knowledge, there has been only one case report of CAS performed in a patient with right-sided aortic arch. ${ }^{10)}$ In that report, the percutaneous approach was performed via the right femoral artery, a 9-Fr balloon-guiding catheter (OPTIMO) was placed in the left CCA, and a Carotid Wallstent was deployed successfully at the level of stenosis under dual protection using the proximal balloon and distal filter protection (FilterWire EZ: Boston Scientific, Natick, Massachusetts, USA). In our case, a 4-Fr modified Simmons catheter was placed in the origin of the left CCA but could not be advanced into the left CCA. Then, the patient underwent CAS using a trans-brachial arterial access with a 6-Fr guiding sheath. We speculated that the catheter could not be advanced into the left CCA via the transfemoral approach because the left CCA in the present case originated from the ascending aorta more proximally to the heart when compared with that of the previously reported case. Therefore, the 4-Fr modified Simmons catheter passed through two steep turns at the top of aorta and the low origin of the left CCA, respectively. This might be the reason why the inner catheter fell into ascending aorta when we tried to advance the catheter to the left CCA. In contrast, the advantage of the trans-brachial approach in this case is that the catheter passes through only one steep turn at the low origin of the left CCA arising from the ascending aorta. In this condition, important points might be to choose an appropriate inner catheter, and how to advance the inner catheter to the CCA. The guidewire should be exchanged for a stiff guidewire at the time of guiding sheath navigation to the CCA. ${ }^{11)}$

Unfavorable complex anatomy and/or congenital anomalies of the supra-aortic vessel take-off may increase CAS procedural difficulty and complications when using the femoral route. On the other hand, the right brachial approach for left CAS in a bovine-type aortic arch or the right brachial 
approach in a type-III aortic arch for CAS appears to be safe and technically feasible. ${ }^{12-14)}$ When the anatomical characteristics of a right-sided aortic arch limits navigation of a guiding catheter into the CCA from the trans-femoral access, placement of a guiding catheter to the CCA from the right brachial access should be considered as an alternative.

\section{Conclusion}

In summary, the report described a case of percutaneous treatment of a pseudo-occlusion of the left ICA accompanied by aortic anomalies consisting of a right-sided aortic arch. CAS via the trans-femoral approach was not successful due to anatomical difficulties. CAS via the transbrachial approach allowed the placement of a 6-Fr guiding sheath to the left CCA and resulted in a successful stenting procedure.

\section{Conflicts of Interest Disclosure}

The authors declare that there are no conflicts of interest.

\section{References}

1) Shuford WH, Sybers RG, Gordon IJ, Baron MG, Carson GC: Circumflex retroesophageal right aortic arch simulating mediastinal tumor or dissecting aneurysm. AJR Am J Roentgenol 146: 491-496, 1986

2) Hastreiter AR, D'Cruz IA, Cantez T, Namin EP, Licata R: Right-sided aorta. I. Occurrence of right aortic arch in various types of congenital heart disease. II. Right aortic arch, right descending aorta, and associated anomalies. Br Heart J 28: 722-739, 1966

3) Cinà CS, Arena GO, Bruin G, Clase CM: Kommerell's diverticulum and aneurysmal right-sided aortic arch: a case report and review of the literature. J Vasc Surg 32: 1208-1214, 2000

4) Cinà CS, Althani H, Pasenau J, Abouzahr L: Kommerell's diverticulum and right-sided aortic arch: a cohort study and review of the literature. J Vasc Surg 39: 131-139, 2004

5) Kuroda S, Kamiyama H, Abe H, Houkin K, Isobe M, Mitsumori K: Acetazolamide test in detecting reduced cerebral perfusion reserve and predicting long-term prognosis in patients with internal carotid artery occlusion. Neurosurgery 32: 912-918; discussion 918-919, 1993

6) Edwards JE: Anomalies of the derivatives of the aortic arch system. Med Clin North Am 32: 925-949, 1948

7) Fu M, Hung JS, Liao PK, Chang CH: Isolated right-sided patent ductus arteriosus in right-sided aortic arch. Report of two cases. Chest 91: 623-625, 1987

8) Floten HS, Rose DM, Cunningham JN: Surgical therapy of a dissecting aortic aneurysm involving a right-sided aortic arch. J Am Coll Cardiol 4: 1058-1061, 1984

9) Haughton VM, Fellows KE, Rosenbaum AE: The cervical aortic arches. Radiology 114: 675-681, 1975

10) Sakamoto S, Shibukawa M, Tani I, Araki O, Oki S, Kiura Y, Okazaki T, Kurisu K: Carotid artery stenting in a patient with right-sided aortic arch with an aberrant left subclavian artery. Acta Neurochir (Wien) 153: 2169-2173, 2011

11) Hayakawa M, Takigawa T, Kamiya $Y$, Tsuruta W, Matsumaru Y: Carotid artery stenting via the transradial approach: a single-center experience. JNET 6: 16-24, 2012 (Japanese)

12) Montorsi $P$, Galli $S$, Ravagnani $P$, Ghulam Ali S, Trabattoni $D$, Fabbiocchi F, Lualdi A, Ballerini G, Andreini D, Pontone G, Annoni A, Bartorelli AL: Carotid stenting through the right brachial approach for left internal carotid artery stenosis and bovine aortic arch configuration. Eur Radiol 19: 2009-2015, 2009

13) Fang HY, Chung SY, Sun CK, Youssef AA, Bhasin A, Tsai TH, Yang $\mathrm{CH}$, Chen CJ, Hussein H, Wu CJ, Yip HK: Transradial and transbrachial arterial approach for simultaneous carotid angiographic examination and stenting using catheter looping and retrograde engagement technique. Ann Vasc Surg 24: 670-679, 2010

14) Dahm JB, van Buuren F, Hansen C, Becker J, Wolpers HG: The concept of an anatomy related individual arterial access: lowering technical and clinical complications with transradial access in bovine- and type-III aortic arch carotid artery stenting. VASA 40: 468-473, 2011

Corresponding author:

Toshiyuki Ohtani, MD, Department of Neurosurgery, National Hospital Organization, Takasaki General Medical Center, 36 Takamatsu-cho, Takasaki, Gunma 370-0829, Japan.

$\triangle$ totani@takasaki-hosp.jp 